\title{
Congress Highlights and Reflections
}

\author{
STANLEY G. BROWNE \\ Secretary-General of the Tenth \\ International Leprosy Congress
}

Highlights and Reflections - not a bird's-eye view gained from the vantage-point of one who has been looking down, but rather from the standpoint, or rather the wriggling point, of one who, like a worm, has been looking up. This summary might therefore be more suitably entitled, "A worm's-eye view of the week's work".

The worm may be a book-worm. Long before the Books of Abstracts saw the light of day, the Secretary-General was worming his way through the 378 abstracts in English, French, and Spanish. Like an earth-worm, too, he tried to ingest the "grounds" of new knowledge and then to evacuate or regurgitate them, adding perhaps a modicum of that indefinable something that enhances fertility and productivity. Now I have to be a kind of hook-worm, hooking on to, and catching hold of, the more important and succulent and nutritious morsels. I hope also now to act as a glow-worm, and to illuminate, however slightly, the obscurity and darkness of turgid scientific sessions.

We will leave the ultimate impact of this Congress to history and to objective discussion in places where leprologists and others foregather in treatment and control programmes, in immunological laboratories where synthetic compounds are prepared, tested, evaluated, of tentimes discarded or tentatively offered for clinical trial-in all these places I hope that the findings of this Congress will be critically appraised and reviewed, and then of fered to those concerned with leprosy in the field and wherever leprosy patients congregate in their expectant thousands.

I shall, therefore, attempt to summarize, however briefly and superficially, the two concurrent sessions that have taken place during the past few days. I must express my indebtedness to the Chairmen who have furnished me with short reports of their sessions.

We begin with the patient suffering from leprosy today. The ordinary physician, in some of our sessions, may seem to have been almost forgotten amid the plethora of laboratory-orientated research reported. Field workers, of course, are primordial and paramount in the actual struggle against leprosy in the individual patient, and I dare to remind those working in laboratories of this fact. But the workers in the field would be the first to admit, when they have the chance to stand back and stare, that they are dependent upon other factors than clinical acumen, accurate observation and full and meticulous recording of the observed data-they are dependent more than ever upon research carried out in the laboratory.

I will juggle with the programme as prepared, so that we may start at the logical beginning and finish at the logical end, glancing briefly at the past and peering tentatively into the future. 


\section{Session 18: Clinical Aspects \\ Co-chairmen: Dr R. D. Azulay, Dr A. B. A. Karat}

The clinical aspects come first in our range of thinking. Gone are the days, fortunately, of long and dreary and oft-times acrimonious discussion and debate over the niceties and the minutiae of classification. Everything now seems to be in reasonable order, or is rapidly becoming so. Thanks to our colleagues engaged in immunological research, things are far more orderly than they were. We can see the differences and the reasons, or partly see the reasons for the differences, between the extreme clinical manifestations of the host-parasite relation. We can see something of the meaning of the immunological spectrum, something of the wonderful orderliness of the pathological reaction to a very curious, almost unique, micro-organism. The clinical aspects of leprosy had a very small part in this Congress, because they are by now generally accepted. There is still much to learn, still much to record by leprologists who have eyes that see and perceive, but all our clinical observations must be subject to the severe arbitrament of independent histological examination. We saw this necessary juxtaposition in the papers and the discussion on indeterminate leprosy where, once again, it was the histopathologist in his laboratory who could suggest or confirm or reject, as he peered through serial sections, looking for indubitable evidence of mycobacterial infection in the tiny nerve fibrils.

And then, one step removed, the neurotropic predilection of this very curious micro-organism challenges both the clinician and the immunopathologist. Why does the organism do this? Why does it do it as it does? Why does it not do the same thing in other tissues? From this question of fundamental pathogenesis, this session proceeded to a study of the loss of sensation, which, of course, is a far more important factor in plantar ulceration than intrinsic motor paralysis.

To remind the clinicians that the last word has not yet been said about the clinical manifestations of leprosy, the question of lepromatous infiltration of the scalp was raised. "Leprosy lesions never occur in the scalp" we have been told. But they do, and when you look hard you can find them, and when you take out pieces of skin, fix, stain, cut and examine them you can find evidence of leprosy in the scalp. Do not, I beg you, repeat unthinkingly and uncritically what you learned from textbooks 30 years ago. Subject once again your clinical observations to the intensive arbitrament of objective histopathological examination. Do not copy statements made ex cathedra and surrounded by a hallowed, cloistered aura from the ancient past. Use your eyes, keep records, investigate histopathologically. Taste sensation has recently been investigated, and the techniques of electromyography are being utilized in the service of the leprosy patient.

\section{History}

Henry Ford, it is reported, once said that all history is bunk. Most remembered history is not only bunk-it is junk. The study of leprosy history, on the other hand, is a fascinating and extremely rewarding hobby, shedding light on the indubitable osteological evidence of leprosy in the ancient world. The buried past, excavated and minutely examined, indicates the spread of leprosy in the Western world in ancient times. Dry bones have indeed come alive under the penetrating eyes of modern osteo-archaeologists. The living counterparts of these long-buried leprosy sufferers, subjected to radioscopy in India and Thailand and South 
America, furnish evidence that the old scourge is still eroding the anterior nasal spine and the alveolar process of the maxilla.

\section{Session 3: Advances in Epidemiology Co-chairmen: Professor M. F. Lechat, Dr R. S. Guinto}

But we must hurry on to the present. Norway, with its wonderful records and its very convincing success story, intrigued us this week with accounts of the national leprosy registry, and the pioneering activities of percipient souls who not only studied leprosy in the laboratory but went into the field to find out more. This success story cannot unfortunately be repeated in other parts of the world for various good reasons, but some of the lessons learned from Norway are, indeed, applicable elsewhere, as we found when we turned our attention to epidemiology. Hansen himself has many worthy followers in many countriesmen who demonstrate a happy combination between laboratory-orientated research and routine field-surveys, supported by meticulously kept records. How much more could we learn if more records of this type were available! The basic principles of epidemiological research today are reinforced by new investigative techniques, such as the lymphocyte transformation test, computer simulation, epidemiometric models, and the rest.

\section{Sessions 5 and 7: Control Chairmen: Dr L. M. Bechelli, Dr D. A. Russell}

Epidemiology is frequently associated, of course, with control, and so it must be in our thinking today. A very interesting series of papers was presented in the session on Control. Historically we have passed through successive phases, with some amount of overlapping: there was the sheer hopeless pessimism of the pre-sulphone days when, despite chaulmoogra and hydnocarpus oils, gynocardate of sodium, Alepol, Graumanyl and many others, including diphtheria toxoid and methylene blue, we could do very little for the leprosy sufferer. Then came the sulphones, and what a wave of journalistic optimism we experienced! And then, sober realism, even pessimism-almost despair. Now, dare I say, some considerable confusion and perplexity. What help can BCG vaccination afford? How soon can we expect a vaccine, a specific vaccine, that will stimulate convincingly the development of an adequate cell-mediated immunity in the individual in danger of succumbing to the risk of lepromatous leprosy? And so, once again, hand in hand, we must go over to the analytical biochemists and the immunologists, to produce a specific vaccine from the highly bacilliferous tissue available from sacrificed armadillos. In the experimentally infected armadillo, with its close simulation of human lepromatoid leprosy, we now have available a model and a source of relatively huge amounts of material for biochemical fractionation and analysis, and immunological research.

Integration of leprosy into the general health services is an ideal to be aimed at-an accepted ideal, sometimes impossible of realization, often attainable if actively pursued. From integration it is a short but necessary step to chemoprophylaxis with acedapsone, as shown in the convincing demonstration in Micronesia and in Papua New Guinea, and in other parts of the world. 


\section{Session 15: Rehabilitation \\ Co-chairmen: Dr O. W. Hasselblad, Dr T. T. Arvello}

From Epidemiology and Control, we pass to Rehabilitation. I find the accent shifting, subtly, from heroic and expensive attempts to rehabilitate a few individuals to serious large-scale efforts at prevention.

Primary patient care, we were reminded, is all-important, but far too of ten such care is either not available or is too little or comes too late. That is the tragedy of leprosy today--we know enough but we are not doing enough. A special rehabilitation team is an ideal scarcely attainable in most parts of the world, but every patient in danger of suffering from the results of peripheral nerve damage should have a minimum of preventive care. There must also be health education for the patient and his community; immobilization of limbs and soft parts in danger of damage from unappreciated traumata; care, especially, for early eye complications and their recognition, even by auxiliary workers; and protective footwear, so that patients with anaesthetic soles may safely go about their business or to their paddy fields or their offices. The importance of training was underlined as one of the most important contributions the Western world can make to the developing countries, to share with them know-how, experience, and staff.

These rehabilitation services must be integrated in to general community services for the handicapped, making no distinction and perpetuating no stigma, so that the leprosy sufferer, whatever the stage of his leprosy, may be accorded the social rehabilitative and surgical services of which he, as a citizen, is in dire need, and to which he, as a citizen, has every right.

\section{Sessions 17 and 19: Advances in Therapy Co-chairmen: Dr K. Ramanujam, Dr J. Languillon}

From Rehabilitation to Therapy. At the last Congress, you may remember, I stuck my neck out and said, "If only we could together apply existing knowledge, it is not beyond the realms of possibility that leprosy could be controlled in this generation and eradicated in the next". After five years, we must sadly confess that we did not apply the then existing knowledge, and now we are confronted with problems on many hands. We had the tools five years ago; perhaps we could have done the job. The tools are now getting blunt, and we are menaced by the problems of drug resistance, ineffective treatment programmes and lack of perseverance on the part of patients and staff. My words of five years ago are not as applicable now as they were then.

What then is the outlook of treatment today? More is known about the effectiveness and the limitations of drugs long used in leprosy-dapsone, the long-acting sulphonamides and clofazimine, in particular. But in sulphone resistance, we have a spectre that looms ever larger across the world of leprosy. Some will object that we now have clofazimine and rifampicin to give the patients harbouring dapsone-resistant strains of Myco. leprae; but many developing countries, trying to "make do" on 6 to 10 Norwegian Crowns per head per year for all medical services, cannot afford rifampicin for the favoured few. Should they spread their resources thinly over large populations, running the risk of irregular treatment, of the development of sulphone resistance; or should we seek something better? We must seek something better, something more effective, more certain, and not very much dearer. Here we enter the possibilities of depot- 
and slow-release drugs, like acedapsone, with the demonstration in the Philippines, in Micronesia and elsewhere, that these are an effective treatment, notwithstanding the low blood levels of sulphone registered in various investigations; and notwithstanding the risk of the development of drug-resistant strains and their emergence in a significant proportion of patients under treatment. More work is needed along these lines.

We now come to rifampicin and the 200 or more different semi-synthetic derivatives of the antibiotic produced by the mould Streptomyces mediterranei. Rifampicin has now been adequately investigated, and has been shown to have distinct bactericidal properties. We must explore ways of modifying the complex and expensive parent molecule to produce new compounds with ever-increasing specificity and effectiveness.

With the help of the controlled clinical trial and adequate laboratory cover, and the possibilities of mouse-footpad investigation, we should be doing more, more quickly and more effectively, in the search for new compounds for leprosy treatment. Somebody mentioned allergic nephropathy following rifampicin therapy, particularly after intermittent régimes, and also the ugly spectre of thrombocytopenia. Rifampicin is not the perfect or final answer; it is the latest, and seems to be, bactericidally speaking. the best. We long for a therapia sterilisans magna-something that will kill all the mycobacteria in the tissues without damaging the host. I commend this thought to the research biochemists.

Combined treatment may be theoretically desirable for the prevention of resistance and for the production of a synergistic effect, but practically, it is virtually impossible in the world with its scattered population suffering from leprosy, its very sketchy, even embryonic, medical services, and its half-trained and inadequately supervised personnel. Here again, the challenge is to do something more and something better.

For the complications of leprosy, particularly lepromatous leprosy, we have in our hands several very effective drugs: clofazimine, thalidomide and the corticosteroids. If only we had a non-teratogenic derivative of an active metabolite of thalidomide, many hundreds of thousands in the world would rejoice. We need something better and more dependable, without the possible teratogenicity and neuropathogenicity of this wonderful drug, thalidomide.

I have mentioned sulphone resistance. This came up once again in the session on Therapy. It is an increasingly serious problem in the world, to be counteracted, in theory at least, by adequate treatment given for an adequate length of time, adequately supervised. Here again, we come up against the hard facts of life-this is not being done throughout the world in adequate measure. We need to do more, and to do it better.

The value of chemoprophylaxis, however, in the prevention of lepromatous leprosy, in reducing its duration when it does eventually appear clinically, and the optimum dose and frequency of administration of the chemoprophylactic-all these are matters still to be determined with accuracy, a challenge to all who are faced by a considerable leprosy problem.

\section{Sessions 13 and 15: Advances in Surgery Co-chairmen: Dr A. G. Warren, Dr E. P. Fritschi}

From medical treatment to surgery. Critical reviews of long-term follow-up of patients who have been operated on have rather tended to counteract the early 
over-enthusiasm apparent in some quarters and, perhaps, a trace of hyperoptimism. These feelings are now replaced by a more sober appraisal. Applied reconstructive surgery may be wonderful and technically very challenging to the surgeon, but ideally it should not be necessary. It is never completely satisfactory; mechanical improvement is not accompanied by comparable functional, aesthetic or sensory improvement. It is very good, but it is not the best. We must prevent -and it is cheaper and better in all ways to prevent than to try to alleviate.

A very fruitful discussion took place on the matter of operating on the acutely inflamed peripheral nerve. Neurolysis of nerves gives sometimes dramatic relief of pain and prevents permanent damage, but there was some divergence of opinion concerning restoration of function in foot-drop. intrinsic and lumbrical paralysis. But the investigations of orthopaedic surgeons and neurohistopathologists-and of neurophysiologists, too-can do nothing but good in advancing knowledge in the fundamental realms of the physiology and pathology of nerves. I regard with great satisfaction this fruitful contact between the research physiologists and the orthopaedic surgeons in the field of leprosy.

With increasing knowledge of structure and function, the possibilities of new and novel procedures of tendon transfer are being actively explored by surgeons in South Africa, West Africa, Europe, the United States and South America.

The human hand is basically primitive phylogenetically, but wonderfully adapted to a whole range of delicate movements, and excuisitively sensitive to a wide range of stimuli. The hand deserves the best of surgery and preventive physiotherapy. And further back still, the individual leprosy patient exposed to the risk of damage to the nerves of the arm and forearm, and the unfortunate sequelae of such damage, should have the best of therapy and of care

\section{Session 5: Ophthalmology Chairman: Dr Margaret Brand}

The sub-section on Ophthalmology provided interest out of proportion to the number of papers presented. Leprosy continues to stand high in the list of causes of blindness throughout the world, especially that due to unappreciated and painless iridocyclitis. All clinicians and physicians should be more aware of the importance of impaired corneal and iris sensation, not necessarily noticed by the patient himself or picked up during the superficial examination made by a para-medical worker. Good equipment is now available, at a reasonable price. Some centres needing a slit-lamp microscope should appeal to a voluntary agency to donate such an instrument and provide for a course of instruction in its use. The simple drugs are still the best-good old-fashioned atropine and good new-fashioned steroids can work wonders-slowly but surely-in preventing blindness. And then, of course, on the theoretical side, sophisticated, electronically operated, substitutes for visual and tactile sensation are being developed, particularly in the U.S.A. - a dream in the future for most people, and not an ever-present help in time of their present trouble.

\section{Sessions 9 and 11: Advances in Pathology Co-chairmen: Dr C. K. Job, Dr D. S. Ridley}

And so to the consideration of the basic pathology of leprosy. Several interesting reviews were given of the histopathology of nerves in leprosy and new 
findings in diseased nerves. The changes in the perineurium and blood vessels leading to invasion of the nerve by inflammatory exudate followed by nerve destruction, were examined in a series of papers, and the similarity of nerve changes in human leprosy and in experimental mouse leprosy was shown.

There were good demonstrations of demyelination and reduction of internodal lengths of individual nerve fibres, isolated from lepromatous nerves. Work with limited but interesting potential was presented concerning nerve graf ts and the restoration of sensation.

But pathology is not what some of us studied years ago in medical school. We have to learn a new language if we would understand the microbiology and immunopathology and experimental therapeutics of today.

\section{Sessions 8 and 10: Advances in Microbiology Co-chairmen: Dr Y. Yoshie, Dr J. H. Hanks}

Encouraging success and progress have been reported in many directions in microbiology. Expanding use of the mouse footpad. for instance, and pyridine extractions, lepromin testing, phenol oxidases and the rest show not only that many people are interested in this aspect of leprosy, but that they are doing good, reportable and reproducible work. Myco. leprae has been shown to undergo limited multiplication in human macrophages maintained in vitro. And cultivation trials in cell-free media are exploring the role of inorganic resources of energy, and of adjuvant factors culled from related mycobacteria and even from the soil. Claims are being made of the successful culture of $M y c($. leprae on sof tagar. Other reported investigations concerned the possible complex life cycle of $M y c^{\prime}($. leprae and the róle of non-acid-fast forms.

Electronmicroscopy, too, is revealing real differences in susceptible hosts, and mycobacteria which, under the light microscope and ordinary staining, seemed very similar, even identical, are shown under the piercing glare of the electron microscope to have certain fundamental differences in surface and internal structure. To jump from the human being to the experimental mouse, some limited extra-cellular growth of Myco. lepraemurium was reported to occur in a liquid medium supplemented by certain compounds and monitored by scanning electronmicroscopy; and also in experimental diffusion chambers incubated in the peritoneal cavities of mice.

These glimpses into the future possibilities of this kind of experimental investigation are of intriguing interest. Certain serological studies showed close antigenic relations between $M y(0)$. lepraemurium and $M y(0)$. avium. These observations give point to further investigations into the whole range of mycobacteria.

\section{Sessions 4 and 6: Advances in Experimental Leprosy Co-chairmen: Dr R. J. W. Rees, Dr S. R. Pattyn}

From the discussion on the microbiology of Myco. leprae, we turned to experimental leprosy; here, too, some tremendously exciting advances were reported, and significant progress is now in prospect. The mouse model is being increasingly utilized throughout the world, and footpad inoculation is routinely used for identification of Myco. leprae, for indications of viability, for immunological studies, for the assessment of drug resistance, for drug screening 
and for many other procedures, such as, for instance, the pathogenesis of leprosy in the tissues, especially nerves. Another experimental model is now available, the neonatally thymectomized rat. This may be an even more reliable model than the mouse footpad, and with standardized techniques, the immunologically suppressed animal is now available for further use and exploitation.

From far-off Korea came the news that the little chipmunk is now being pressed into service for the cause of leprosy, being itself susceptible to experimental infection with Myco. leprae. Progress was reported in the systemic infection of the nine-banded armadillo, and more and more data are accumulating concerning infection and infectivity, lesions in the skin, the nasal mucosa, the nerves, and the deep organs. The rich source of $M y c^{\circ}$. leprae now available in quantity must be used speedily for fractionation, biochemical analysis, immunological investigations and, hopefully, therapeutic trials. Possible routes of infection, both natural and experimental, are being currently investigated on this model.

From the nose of the armadillo to the nose of the human being is but a small step, and half-forgotten studies stretching back many years are now being confirmed by painstaking specialist clinical examination backed by convincing histopathological demonstration. Once again, I would underline the importance of fruitful contact between the examining clinicians and the investigative laboratory.

The relation of these investigations to the transmission of leprosy, the site of exit and perhaps also the site of entry, give point to the need for clinical observations by field workers. The nose may very rarely be bacteriologically positive before the skin. yet it may be the last to shed viable and non-viable bacilli from the extensive infolded mucosa covering the septum and the inferior and middle turbinates. The output of $M y c o$. leprae every day from such a nose is similar to that of patients with open tuberculosis of the lung, and is indeed colossal. Millions of $M y C^{\prime}(\mathrm{O}$. leprae, a high proportion of which may be shown to be viable when injected into the mouse footpad, are being shed daily from the nasal mucosa of unsuspecting patients.

The persistence of viability of these $M y c o$. leprae-up to nearly two days after discharge from the nasal mucosa-is a very interesting and potentially very important point when we consider the duration of infectivity of patients and re-examine the possible importance of fomites in the persistence of the leprosy endemic, or the occurrence of cases of leprosy many miles from the potential source of viable mycobacteria. There is experimental evidence that $M y c^{\circ}($ ). leprae in the ambient air may enter mice by a route not yet convincingly demonstrated, but possibly the respiratory tract.

\section{Session 2: Advances in Experimental Therapeutics Co-chairmen: Dr C. C. Shepard, Dr M. F. R. Waters}

This section was concerned with the development of new drugs and the procedures to be followed in the biochemical development of new therapeutic agents, particularly the identification of enzyme systems that might at some point be susceptible to a drug that could be termed, loosely, lethal. Dehydropterocete and the dihydrofolate systems with the de novo folate pathways, are of extreme interest and importance in this connection. We have to hit Myco. leprae hard, and at one or two susceptible points in its multiplication and development, so that the 
human being suffering from leprosy may have a better hope of rapid cure. He is not interested in the morphology of $M y c^{\circ}($ ). leprue or in their concentration. He wants to be cured of what he is suffering from, and he does not want to remain ostracized. Hence the importance of these rather sophisticated laboratory investigations, and of the trial of acedapsone which was also reported in this section.

\section{Sessions 12, 14 and 16: Advances in Immunopathology Co-chairmen: Dr O. K. Skinsnes, Dr T. Godal}

Lastly, we come, after this brief and all-too-superficial worm's-eye view of this week's work, to those extremely interesting and important sessions on immunopathology -in many ways the most important of the week - the best reserved for the last. Since the London Congress in 1968, new methods have been developed that make it possible actually to measure cell-mediated immunity to leprosy infection in vitro. Studies undertaken throughout the whole of the clinical and histopathological spectrum of leprosy lend strong support to this conception of a unique host-determined immunological defect in lepromatous leprosy. These matters are being actively studied in many places, but particularly in the A.H.R.I. Institute, sponsored by the Scandinavians, in Addis Ababa. There is also a non-specific depression of cell-mediated immune processes in active lepromatous leprosy with both humoral and cellular aberrations. The early observations suggesting a link between hepatitis-associated antigenaemia and lepromatous leprosy have not been substantiated, to the surprise perhaps, of many participants. Nor is there any specific evidence today of the importance of a genetic factor in leprosy, though some reports suggest that this may be so. Cell-mediated immunity to Myco. leprae is an important mechanism of tissue damage in reversal reaction, and circulating immune complexes have been reported in erythema nodosum leprosum. This observation reinforces the real dangers inherent in the use of homologous lymphocytes for the treatment of human patients with leprosy: we must always remember our ethical responsibilities as we pursue laudable therapeutic aims and investigations.

Preliminary studies utilizing the lymphocyte transformation test and other new techniques give hope that the search for an effective vaccine may now progress more rapidly; it may not be "just around the corner"-and the corner may be longer than we hoped-but the possibility is there.

Before the worm turns and wriggles off to his secretarial burrow, he would emphasize that at the centre and heart of the International Leprosy Association lies the whole raison d'être of this Congress-the pursuit of new knowledge so that the individual sufferer from leprosy may be helped and the community spared the ravages of this disease. Amid the plethora of exciting new investigations, the leprosy sufferer provides the personal challenge to the research worker and the clinician. New knowledge must be incorporated into new dimensions of helpfulness towards the patient and the community of which he should continue to be an integral part.

We neglect at our peril the social aspects of this disease complex we are all trying, in our different ways, to overcome. 